\title{
Using confidence factors to share control between a mobile robot tele-operater and ultrasonic sensors
}

\author{
David Adrian Sanders \\ School of Engineering \\ University of Portsmouth \\ Portsmouth, UK \\ david.sanders@port.ac.uk \\ Alexander Gegov \\ School of computing \\ University of Portsmouth \\ Portsmouth, UK \\ alexander.gegov@port.ac.uk
}

\author{
Benjamin John Sanders \\ Peter Symonds College \\ Owens Road \\ Winchester, UK \\ ben.sanders@serg.org.uk
}

\author{
David Ndzi \\ School of Engineering \\ University of Portsmouth \\ Portsmouth, UK \\ david.ndzi@port.ac.uk
}

\begin{abstract}
A system is presented that shares control between ultrasonic sensors, a tele-operator and a mobile robot. The mobile robot can be directed by the tele-operator, or by ultrasonic sensors, or they can share control. The mobile robot system can change direction if there are obstacles ahead or if it is helpful. Sharing control allows a human tele-operator to drive efficiently and safely. Controller gains are set automatically for a human tele-operator and the ultrasonic sensor system by calculating a confidence factor for the mobile robot tele-operator. The ultrasonic sensor system can assist a human tele-operator in driving the mobile robot to offset for shortcomings, for example the tele-operator may not be able to see the mobile robot or the human tele-operator may be tired. Finally, some testing is described to validate the proposed methods.
\end{abstract}

Keywords- Mobile robot; Tele-operator; Confidence factor; Shared-control.

\section{INTRODUCTION}

There are numerous systems described in the literature for helping human tele-operators to work in hazardous or remote environments [1-9]. Research has explored the way that teleoperators can cooperate with mobile robots [10-12]. If the teleoperator is some distance from a mobile robot then time delay may cause problems [13] and reduce efficiency [14]. Teleoperated controllers tend to trade stability margin for clarity [15]. Some approaches have attempted to overcome time delays [3, 16-19].

Unstructured environments can make operation more difficult for mobile-robots [20-26]. Tele-operators control and directed their mobile robots from a place of safety [27]. A wheeled robot is studied in this paper because that is still the most common type [22-24, 28].

The master control input device for a mobile robot has often been a low current joystick [22-24]. A mobile robot draws a higher current to drive the motors. The robot is remotely controlled using a trailing umbilical connection or radio link.
Tele-operation has especially been explored for maintenance and operation in hostile, foul or dangerous environments [27]. A human tele-operator is usually better at driving than a computer so the systems described here attempt to assist a human tele-operator. A wheeled mobile robot base with a manipulator-arm attached to it can achieve complex manipulation and handling tasks [29-31].

In emergencies, efficient interaction between the mobile robot and a human tele-operator can make all the difference. This research explores that collaboration and interfacing.

Tele-operation tends to be open-loop. A human teleoperator indicates a desired direction and their robot attempts to travel on that bearing. Differences between the wheels on the mobile robot or different responses to a variety of gradients and surfaces can disturb the path. Tele-operators need to react to the disturbances and correct the mobile robot path.

Tele-operation, telerobotics and telepresence are explained in two significant papers by Sheridan [2] and [3].

Unpredictable situations can happen [35] that might affect a mobile robot tele-operator and mobile robot operation [36]. Collaboration between the sensor systems on board the mobile robot and a human tele-operator [37] can help the tele-operator conduct difficult tasks more efficiently [38].

Kuniaki [39] presented a collaborative system and Macharet [40] presented a tele-presence system using ultrasonics to show the bearings to targets using triangulation.

Methods described here allow intimate collaboration between ultrasonic sensors and a mobile robot tele-operator as a result of merging human tele-operator commands with data from the mobile robot sensors. A combined control architecture is explained in [41] that promotes cooperation between a robot and tele-operator. In [42], combined control is described that improves performance and reduces workload by providing feedback from an automated process. Autonomous systems can assist a tele-operator to improve reduce workload 
and improve safety for vehicles [43], [44],. Satti [45] described combined control employing computer-brain interfaces. A controller is presented in [46] that controls quadcopters and that can avoid collisions and fly the copters in formation.

In helping a tele-operator, commands may be constrained but a mobile robot may not be able to generate new commands [9]. Haptics allow commands to be given directly to a mobile robot using a haptic-device [47]. A human tele-operator and the sensors can provide commands simultaneously and in that case they can be fused using specified ratios [48]. How authority is distributed is significant for efficient co-operation. Numerous interfaces might be employed [49-55]

In [56], Carlson et al described methods to predict a direction of travel and change the control signals to make a robot move in that direction. Experimentation provided the parameters [57] and dynamic-distribution adjusted the distribution of control in real-time [58]. In [59], the weights were changed after evaluating the commands form the human operator.

Methods to assist a mobile robot tele-operator in complex and changing environments are introduced in this paper. By combining suggestions from sensors and commands from a human tele-operator, both the tele-operator and the sensors cooperate to produce safe movements. The sensor system knows the mobile robot status and then directs the robot to turn. The mobile robot moves in that direction but avoids obstructions along the way.

Section II is a description of the mobile robot and sensors. The controller is described in Section III and shared control in Section IV. Section V discusses the results and VI summarises work. Some future work is suggested in VII.

\section{MOBILE ROBOT AND SENSORS}

This research used a Bobcat II Mobile robot [9, 13] consisting of: inputs from a tele-operator and sensors, the robot base, ultrasonic sensors used to avoid obstacles, and the shared controller. Ultrasonic sensors provided ranges to obstacles ahead of the robot. Data from the sensors were processed by a computer that adjusted the speed and direction of the robot base.

\section{A. The robot}

The mobile robot is shown in Fig. 1. The mobile robot base had four wheels. Two large driving wheels at the front and two casters at the back. Each driving wheel was attached to a motor and could be driven independently.

The mobile robot was steered by changing the current sent to each wheel motor. The mobile robot could turn on it's center-of-rotation [60]. If $\mathrm{V}$ is mobile robot linear velocity, $\omega$ is angular velocity and $\phi$ is direction, then velocity at the center of mass of the robot base is

$$
\mathrm{V}_{\mathrm{c}}=(\mathrm{V}, \phi, \omega)
$$

The kinematic model is explained in [61].
Independently driving the wheels produced orientation and movement because the driving-wheels of the mobile robot were on the same axis.

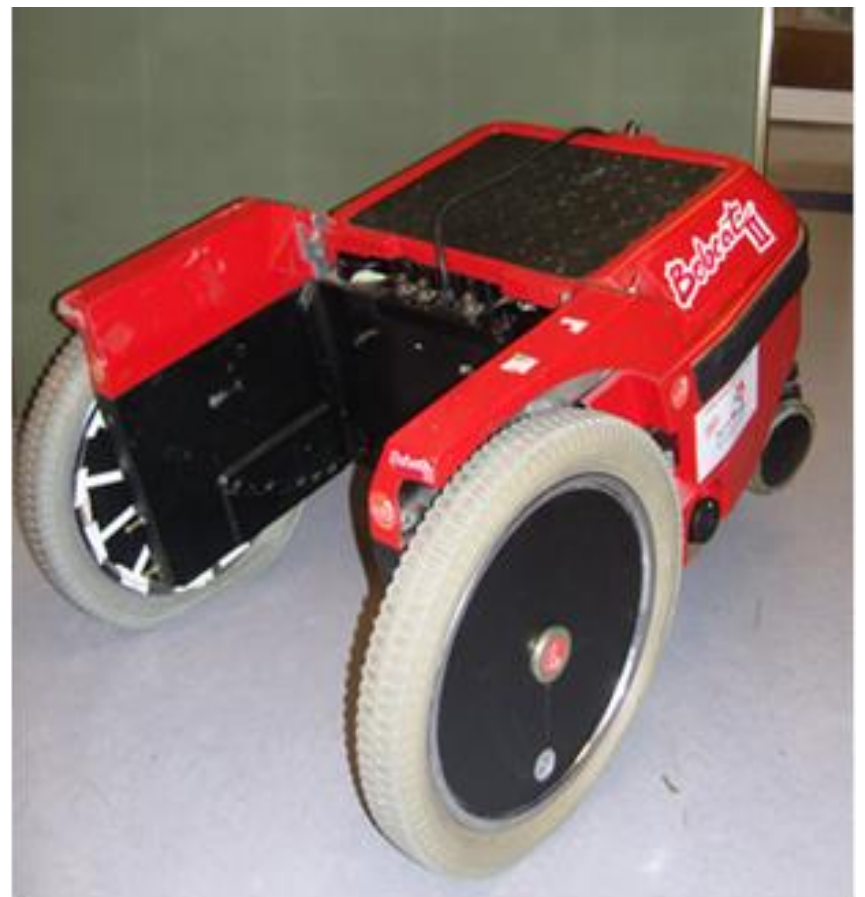

Fig. 1 The Bobcat II mobile robot base

\section{B. Ultrasonics}

Ultrasonic sensors detected obstacles ahead of the mobile robot. The transmitters needed a $3 \mathrm{~m} \mathrm{~s}$ pulse to achieve the highest output. Long pulses held more energy and could detect obstacles at longer ranges. If the speed of sound is assumed to be $330 \mathrm{~m} / \mathrm{s}$... then a $3 \mathrm{~m}$ s sound pulse is $0.99 \mathrm{~m}$ long. Permitting a pulse to exit from it's transmitter, rebound back from an obstacle and reappear back at a receiver, suggests $0.5 \mathrm{~m}$ is a minimum range for a $3 \mathrm{~ms}$ pulse. The work needed ranges that were closer and so various shorter pulse lengths were used.

Obstacles appeared and disappeared when the robot travelled about and it was sometimes challenging to lock on to a target. Ultrasonics were noisy and returned some misreads. Misreads were filtered out to improve reliability. Histogramic In-Motion Mapping was used. Volumes ahead of the mobile robot were separated in to a 3 sector grid and stored in an array: far, middle and near. Ultrasonic transducers were fixed so that they overlapped and covered the area ahead.

Array elements were incremented by five if they contained an obstacle. Array elements that did not contain an obstacle were decremented by 1 .

Fig. 2 illustrates beam patterns for two ultrasonic sensors. 


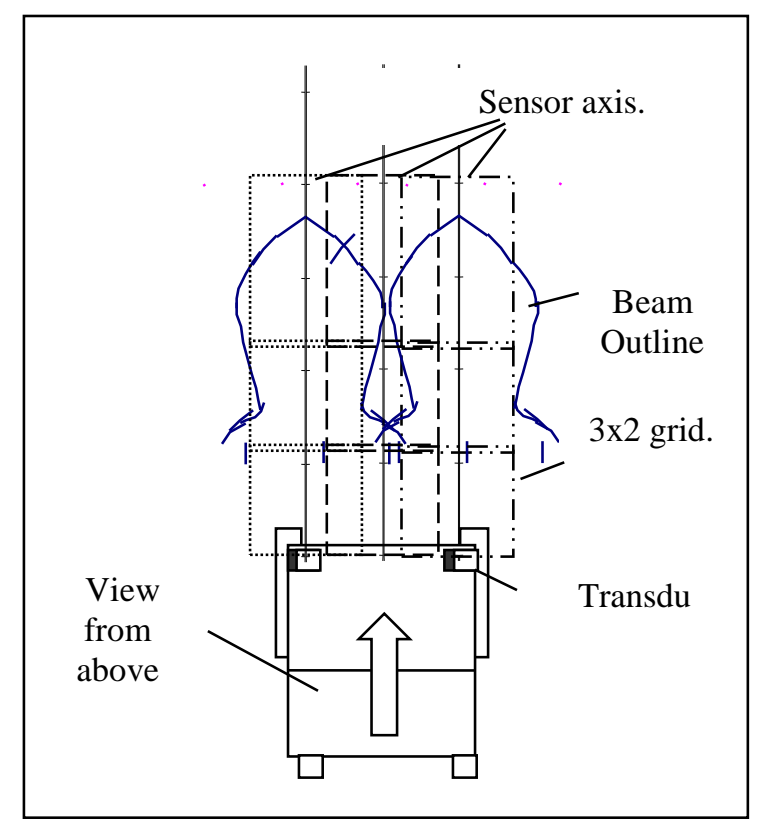

Fig. 2. Overlapping beams from two ultrasonic sensors to create an array.

Arrays had a minimum quantity of 0 and maximum of 15 . Figure 3 symbolizes a 3-element histogrammic depiction of a local environment. An obstacle in the $3^{\text {rd }}$ element is making it increase.

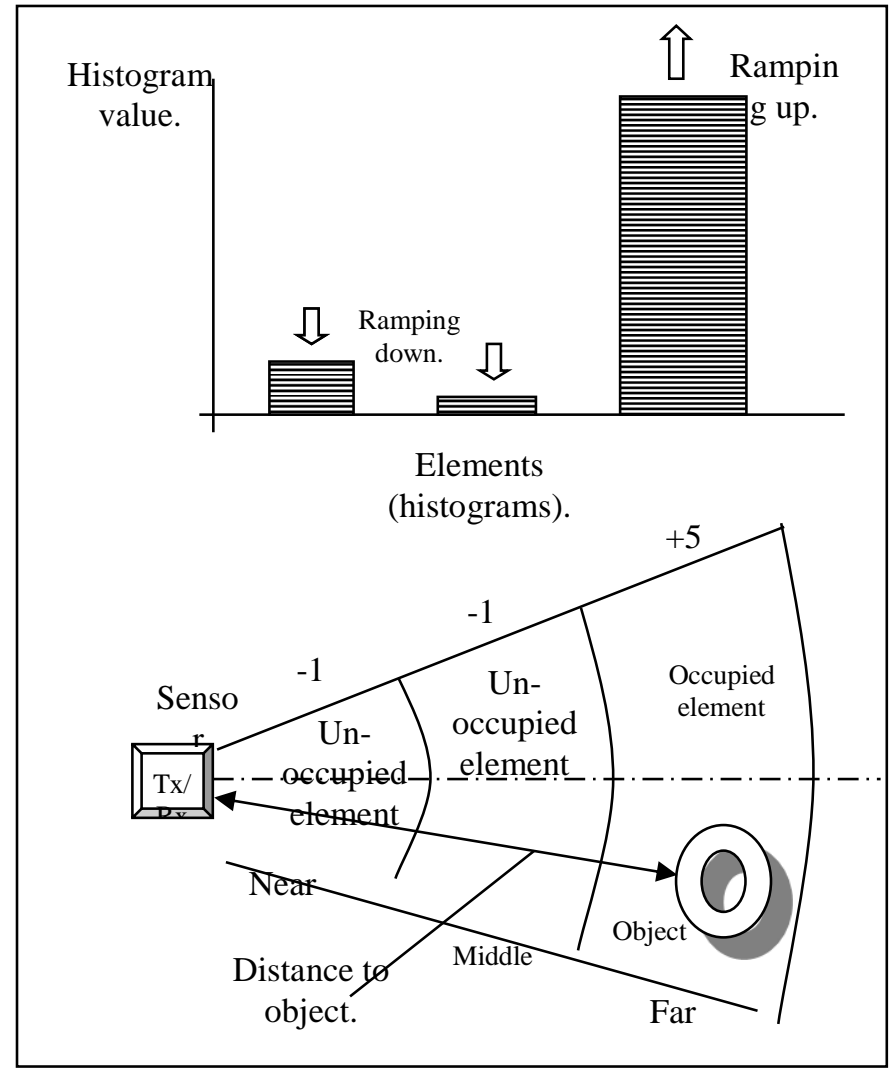

Figure 3. A representation of the sensor histograms. 3-element histogrammic depiction of a local environment. An obstacle in the 3rd element is making it increase.
An obstacle within a grid element caused the element to rapidly increase in value to the highest value. Arbitrary misreads within the other elements increased values briefly, but they were decrimented during every system update period. If the obstacle relocated to another element, then that element rapidly increased in value and the previous one reduced in value. Reliable ranges were acquired within $0.5 \mathrm{~s}$.

\section{CONTROL}

A controller drove the mobile robot following commands from a tele-operator and the sensor system automatically avoided obstacles in the mobile robot path.

\section{A. Controller}

Angular velocity and linear velocity of the mobile robot were considered. The mobile robot followed a desired direction and linear velocity when the mobile robot was at an arbitrary heading angle, as shown in Fig. 4.

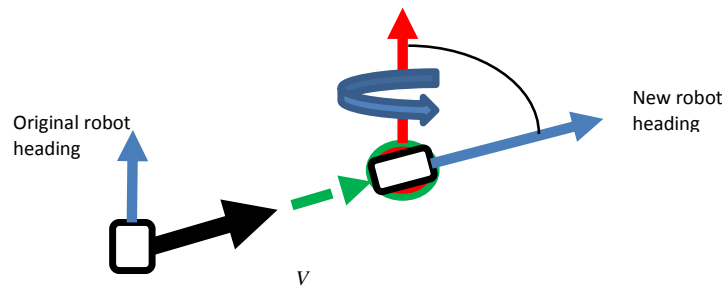

Fig. 4. Controller design.

The control law to track the target position for the mobile robot's linear velocity, $V_{\mathrm{r}}$ was:

$V_{\mathrm{r}}=\mathrm{V}_{\mathrm{M}} \times \mathrm{D} / \mathrm{D}_{\text {Des }}$ when $|\mathrm{D}|<\mathrm{D}_{\text {Des }}$

and

$V_{\mathrm{r}}=\mathrm{V}_{\mathrm{M}} \times \mathrm{D} / \mathrm{D}_{\text {DesSp }}$ when $|\mathrm{D}|>\mathrm{D}_{\text {DesSp }}$

where $V_{\mathrm{M}}$ is the maximum speed of the mobile robot, $\mathrm{D}$ is a vector from the mobile robot joystick, and $\mathrm{D}_{\text {Des }}$ is the demanded speed. If the vector from the mobile robot joystick is greater than the sensor range, the mobile robot moves at the desired speed.

The mobile robot's heading changes during traveling. To track the mobile robot's heading, the control law for the mobile robot's angular velocity, $\omega_{r}$, is defined as:

$\omega_{r}=\omega_{\mathrm{M}} \mathrm{x} \Delta \theta / \theta_{\mathrm{D}}$, when $|\Delta \theta|<\theta_{\mathrm{D}}$

and

$\omega_{r}=\omega_{\mathrm{M}} \mathrm{x} \Delta \theta /|\Delta \theta|, \quad$ when $|\Delta \theta|>\theta_{\mathrm{D}}$ 
Where $\theta_{D}$ is the desired heading, $\omega_{M}$ is the maximum value of the mobile robot's angular velocity and the direction of the mobile robot is expressed as a mobile robot heading error, $\Delta \theta$.

If the mobile robot's heading error is greater than a buffer angle, the mobile robot turns. If the mobile robot's heading error is less than a buffer angle, the control law adjusts angular velocity to track the desired heading.

\section{B. Avoiding obstacles}

The omnidirectional mobility of the mobile robot made obstacle avoidance easier. A vector represented speed and direction. A repulsive force was generated if the mobile robot drove near to an obstacle and the mobile robot steered away from the object (Fig. 5).

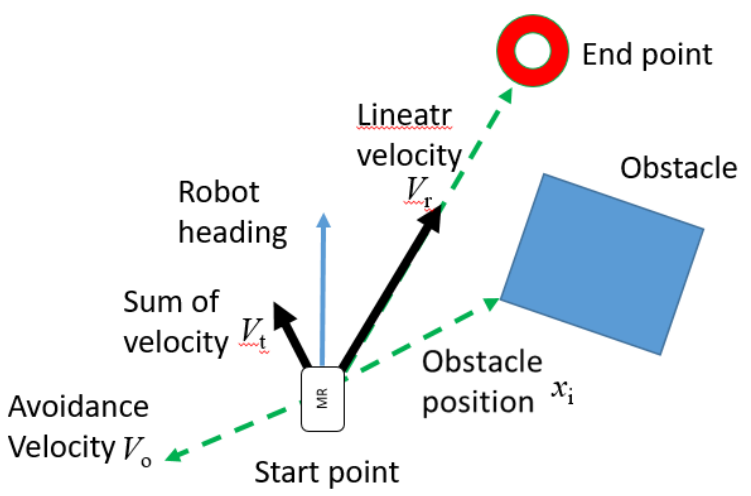

Fig. 5. A repulsive force was generated if the mobile robot drove near to an obstacle and the mobile robot wassteered away from the object.

The avoidance velocity, $\mathbf{V}_{\mathrm{o}}$ was:

$\mathbf{V}_{\mathrm{O}}=a \sum_{\mathrm{I}}\left[\left(\mathrm{D}_{\mathrm{Ms}^{-}}\left|\boldsymbol{x}_{\mathrm{i}}\right|\right) / \mathrm{D}_{\mathrm{s})}\left(-\boldsymbol{x}_{\mathrm{i}} /\left|\boldsymbol{x}_{\mathrm{i}}\right|\right]\right.$

where $D_{s}$ was a safe distance, $x_{i}$ were vectors to represent objects ahead of the mobile robot, and $a$ was a constant. Ultrasonic sensors detected the positions of obstacles, $x_{i}$,

The resultant obstacle velocity, $\boldsymbol{V t}$, was:

$$
\boldsymbol{V}_{t}=\boldsymbol{V}_{r}+\boldsymbol{V}_{o}
$$

where $\boldsymbol{V}_{o}$ was an avoidance velocity that the system generated, $\boldsymbol{V}_{r}$ was linear velocity produced by destination seeking, and, $\boldsymbol{V}_{t}$ was the resultant velocity. $\boldsymbol{V}_{t}$ avoided obstacles but did not change the heading of the mobile robot much.

\section{SHARED CONTROLLER}

Shared-control combined sensor system commands and commands from the tele-operator to improve driving.

A tele-operator could generally control a mobile robot safely but the sensors were more accurate and repeatable. The systems gave autonomy to the human driver and used their skills when possible but intervened if necessary to avoid obstacles.

When the mobile robot operated in varying and complex environments, then the system provided better decisionmaking. The shared and combined-control architecture is shown in Fig. 6.

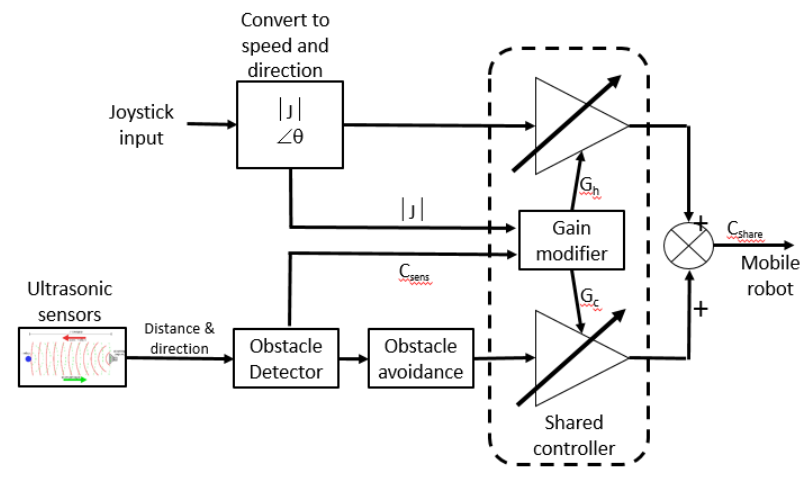

Fig. 6. Shared and combined control.

The shared and combined control extends work described in [55]. It allowed convenient and safe maneuvering of a mobile robot.

The architecture combined a joystick input and shared it with sensor inputs. The tele-operator controlled the mobile robot using a joystick and could usually see the mobile robot. The mobile robot sensor system avoided obstacles and ensured safety when the robot moved.

Current to the mobile robot motors were generated by both a tele-operator and sensors. When obstacles were far away, a human tele-operator didn't need assistance. In environments with many objects or objects near to the mobile robot, the system reduced or inhibited commands from the joystick given by the tele-operator so as to avoid collisions.

The combined-control gains from a tele-operator and sensors changed as the mobile robot moved around. The resultant control command, $\boldsymbol{C}_{\text {share }}$ (Fig. 6) was:

$C_{\text {share }}=G_{h}|\jmath|+G_{c} \boldsymbol{u}_{c} \quad G_{h}, C_{\text {sens }} \in[0,1]$

where $C_{\text {sens }}$ was a range to an object and $|\mathrm{J}|$ was the input from the joystick. $C_{\text {share, was added to the weighted tele- }}$ operator joystick input multiplied by a weighted gain $G_{h}$, and a weighted output from the autonomous controller, $G_{\mathrm{w}}$ was the weighted gain. Confidence-factors established the gains. The system considered the Confidence factor of the tele-operator to determine a tele-operator gain as in (13). 


\section{A. Avoidance Confidence}

Confidence of the tele-operator was estimated. The Confidence Factor was made up of three Factors. An Avoidance Factor was set to represent the ability of a teleoperator. Tele-operators were given a lower Confidence Factor when the mobile robot moved closer to an object. $E_{a v}$, the avoidance-factor, was

$$
E_{a v}=\left|x_{0}\right| / \mathrm{D}_{\mathrm{Sa}}
$$

where $D_{S a}$ was a constant representing a cautious and safe range and $x_{o}$ is the shortest distance between an object and the mobile robot. If the powered mobile robot is further away from an object than $\mathrm{D}_{\mathrm{Sa}}$, then the tele-operator was given a greater confidence rating in driving the mobile robot. If a mobile robot was at a distance less than $\mathrm{D}_{\mathrm{Sa}}$ from an object, then confidence decreased.

\section{B. Safety Confidence}

The Safety Factor denoted the ability of the tele-operator to safely drive a mobile robot. If the mobile robot was operating at low speed, the tele-operator was assumed to be more confident. A Confidence Factor for safety $E_{\text {safe }}$ was:

$$
E_{\text {safe }}=\left\{1-\left(\left|V_{\mathrm{h}}\right| /\left|V_{\mathrm{TH}}\right|\right)\right\}^{q} \text {, for } q<1 \text {. }
$$

where threshold $V_{T H}$ is the fastest linear velocity that a user is permitted to drive a mobile robot and $V_{h}$ was the user's command linear velocity from their joystick. To assign a greater Confidence Factor at low speed, it is projected by means of an exponent, $\mathrm{q}$ (where $\mathrm{q}<1$ ).

\section{Assistance Confidence}

Tiredness and time were important. If a human teleoperator controlled a mobile robot continuously, the teleoperator was liable to grow tired. In that case, their Confidence Factor reduces. A tele-operator was more likely to be alert and awake at the start of a day. Joystick control for an entire day was monitored. Engagement time, $E_{t e}$, is

$E_{t \mathrm{e}}=E_{t \mathrm{e}-1}+\left(1 / T_{\mathrm{a}}\right)$, if tele-operator is rested

$E_{t \mathrm{e}}=E_{t \mathrm{e}-1}-\left(1 / T_{\mathrm{a}}\right)$, if tele-operator is tired

where $T_{\mathrm{a}}$ is the time that a tele-operator has been driving a mobile robot. If a tele-operator actively controlled a mobile robot then estimation slowly dropped. If a tele-operator rested, then estimation increased.

\section{Overall Confidence}

Control gains $\mathrm{G}_{c}$ and $\mathrm{G}_{h}$ and the overall Confidence Factor were:

OverConFact $=E_{t} \times \max \left(E_{\text {avoid }}, E_{\text {safety }}\right)$

$$
\begin{aligned}
\mathrm{G}_{h} & =\text { OverConFact } \\
\mathrm{G}_{c} & =1-\text { OverConFact }
\end{aligned}
$$

When both safety and avoidance confidence factors were high, a tele-operator would drive their mobile robot smoothly. When avoidance was high, the mobile robot was far away from objects in it's path and the tele-operator had complete control of their mobile robot. When safety estimates were higher, the speed of the mobile robot was limited.

\section{RESULTS}

Experiments were undertaken to validate the methods.

\section{A. Simulation}

Simulation validated the mobile robot shared-control. The input from the joystick was fixed to steer to a target destination. Speed was set to $1 / 2$ speed.

An example of a trajectory is represented in Fig. 7.

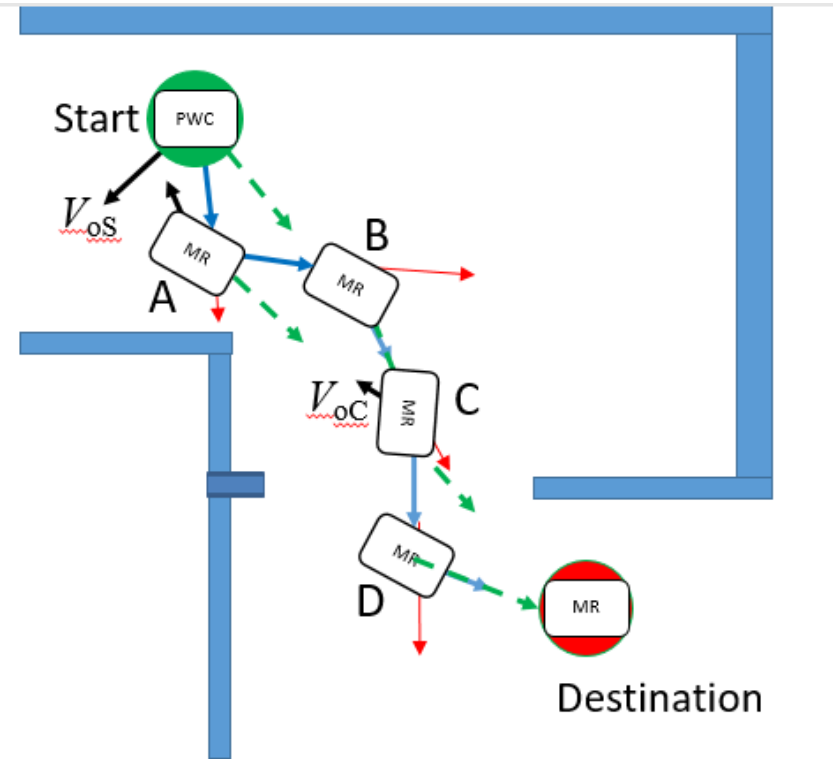

Fig. 7. Simulation experimentation and testing.

Figure 7 shows the mobile robot's heading and position at numerous instants in time. At the start position, the simulated mobile robot was facing right. The the direction to a target destination is represented by a dotted line, that is the input from the joystick. A sensor on the left of the mobile robot detected a wall and guides the mobile robot away. The additional solid line indicates the direction of motion; towards Point A from the Start. As the mobile robot moves towards Point A, the ultrasonic sensor on the right of the mobile robot detects a wall below the mobile robot and turns the mobile robot left to avoid it. Once in free space Vo, reduces to zero 
and the mobile robot turns and moves towards the target destination, guided by the joystick. At Point $\mathrm{C}$ the sensor on the left of the mobile robot detects a doorway and VoC grows so that the mobile robot steers right to avoid the doorway edge.

At Point D and through the doorway, the mobile robot is safe. No obstacles are detected ahead of the robot. The robot can turn and move towards the target.

The simulated mobile robot did not collide with any obstacles and safely approached the target destination.

Values for the Confidence Factors were:

$\begin{array}{ll}\text { Start } & 0.4 \\ \text { A } & 0.6 \\ \text { B } & 1.0 \\ \text { C } & 0.7 \\ \text { D } & 1.0\end{array}$

The Confidence value for avoiding obstacles was significant when objects were detected, so tele-operator confidence was decreased when objects were detected. The mobile robot reduced speed. The sensors partially controlled the mobile robot until it was in open space and safe. The shared and combined control meant that the mobile robot obeyed joystick commands to move towards a destination while avoiding obstacles.

The commands to the robot motors was an amalgamation of a simulated input from a sensor system and simulated joystick input (13). Controller gains for the sensor system and tele-operator produced speed and steering commands for the simulated mobile robot. The simulated mobile robot did not crash.

\section{B. Experimenting with a mobile robot}

Volunteer tele-operators at Portsmouth maneuvered the mobile robot past obstacles to drive to a target destination. Tele-operators controlled the mobile robots using joysticks.

Trajectories were recorded using a camera and a typical mobile robot and Fig. 8. Shows a typical path. Locations for the mobile robot and the associated headings are shown at six positions. The mobile robot could easily turn and was able to spin on it's axis if required, before driving in a selected direction in an attempt to maintain a desired heading. That allowed tele-operators to concentrate on steering. They did not have to concentrate on avoiding obstacles.

The ultrasonic sensors helped tele-operators to control their mobile robots using shared and combined control. The mobile robot did not collide and safely reached the target destination.

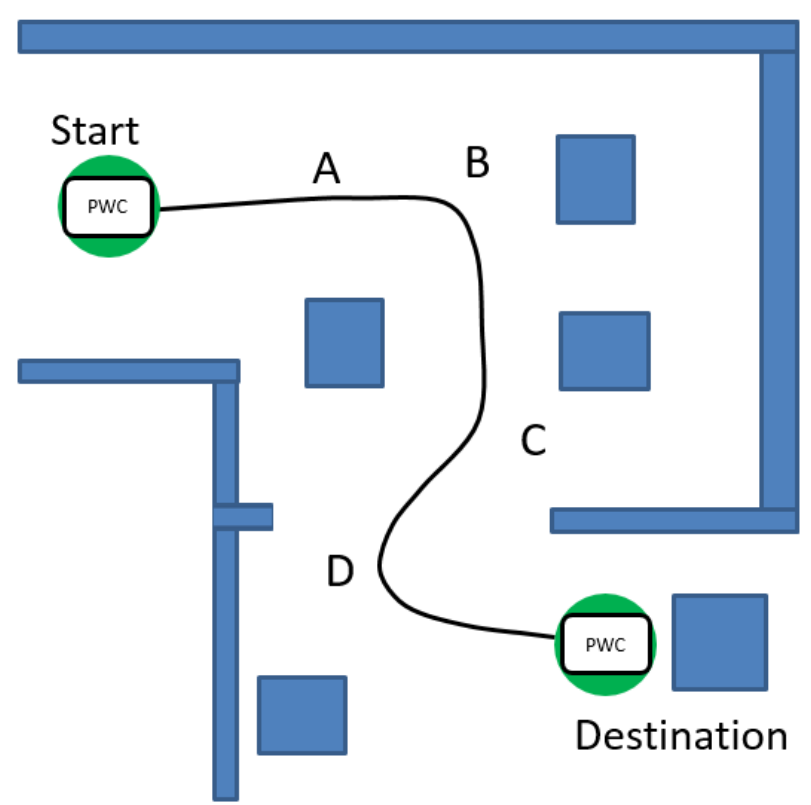

Fig. 8. Recorded trajectory of mobile robot experiment.

Recorded values of Confidence Factor for the experiment were:

$\begin{array}{ll}\text { Start } & 0.0 \\ \text { A } & 0.07 \\ \text { B } & 0.69 \\ \text { C } & 0.69 \\ \text { D } & 0.71\end{array}$

Control of the mobile robot was a combination of inputs from the tele-operator's joystick and from the sensors (13). Control gains for both the tele-operator and the sensors dictated the speed of the two motors and therefor the direction that the robot moved in.

Tele-operator Confidence reduced when obstacles needed to be avoided and the mobile robot did not collide with anything.

If a tele-operator slowed the mobile robot then it became safer and so that tele-operator had a higher authority. Sharedcontrol allowed the mobile robot to move away from objects while following instructions from a tele-operator's joystick.

\section{DISCUSSION AND CONCLUSIONS}

Shared-control was implemented on a mobile robot. A teleoperator was in control of the mobile robot unless sensors needed to assist, for example to avoid an object.

The work did not deal with objects above or below the volume that could be detected by the ultrasonics in the way that they were set up on the robot. 
The mix of the input from sensors and tele-operator were calculated using Confidence Factors. They were established using evidence from the sensors such as: range from the mobile robot to an object; how long the mobile robot operator been driving; etc.

Human tele-operators controlled the mobile robot more safely when assisted by the sensors. Experimental results showed that the shared-control method was safe.

An optimal mix of human verses autonomous control exists for different mobile robot tele-operators in various conditions, for example whether a tele-operator is tired. The most favorable mix changed with human experience and skill.

\section{FUTURE WORK}

The static ultrasonic sensor array is limiting ongoing work and a scanning device has been created at Chailey Heritage (by Martin Langner). Future research will use that device as it is smaller, covers a bigger volume and range and position can be detected more accurately.

Different AI methods are being investigated at Portsmouth [43-52] but they are tending to be more complicated. It is expected that a simple microcontroller and a scanning sensor will be all that is required in future.

\section{REFERENCES.}

[1] D.A. Sanders, The modification of pre-planned manipulator paths to improve the gross motions associated with the pick and place task, ROBOTICA, vol. 13 pp.77-85. 1995.

[2] T.B. Sheridan, Tele-operation, telerobotics and telepresence: A progress report, Control Eng. Pract. 3(2), pp 205-214. 1995.

[3] T. Sheridan, Humans and Automation: System Design and Research Issues. John Wiley and Sons. 1995.

[4] D.A. Sanders, Comparing speed to complete progressively more difficult mobile robot paths between human tele-operators and humans with sensor-systems to assist. ASSEMBLY AUTOM, vol. 29, no. 3, pp. 230-248. 2009.

[5] D.A. Sanders, M. Langner $M$ and G.E. Tewkesbury, Improving wheelchair-driving using a sensor system to control wheelchair-veer and variable-switches as an alternative to digital-switches or joysticks. IND ROBOT, vol. 37, no. 2, pp. 157-167, 2010.

[6] D.A. Sanders and A. Baldwin, X-by-wire technology, Total Vehicle Technology Conference, pp. 3-12. 2001.

[7] D.A. Sanders, Controlling the direction of walkie type forklifts and pallet jacks on sloping ground. ASSEMBLY AUTOM, vol. 28 (4), pp 317-324. 2008.

[8] P. Fiorini and R. Oboe, Internet-based telerobotics: Problems and approaches, Proc' of ICAR'97, Monterey, CA, pp. 765-770. 1997.

[9] J.P. Richard, Time-delay systems: An overview of some recent advances and open problems, Automatica vol. 39, pp 1667-1694. 2003.

[10] D.A Lawrence, Stability and transparency in bilateral tele-operation, IEEE Trans. Robot. Autom. Vol. 9 (5). 1993.

[11] W. Kim, B. Hannaford and A. Bejczy, Force reflection and shared compliant control in operating telemanipulators with time delay IEEE Trans. Robot. Autom. vol. 8(2), 176-185. 1992.

[12] G. Niemeyer and J.J Slotine, Stable adaptive tele-operation. IEEE J. Ocean. Eng. vol. 16(1), 152-162. 1991.

[13] G. Niemeyer and J.J Slotine, Towards force reflecting tele-operation over the Internet, Proc' of the IEEE Int' Conf' on Robotics and
Automation, Leuven, Belgium (ICRA'98), pp. 1909-1915. 1998.

[14] E. Slawinski, V. Mut and J.F. Postigo, Tele-operation of mobile robots with time-varying delay. Robotica, vol 24, pp. 673-681. 2006.

[15] B.L. Luk, D.S. Cooke, S. Galt, A.A Collie and S. Chen, Intelligent legged climbing service robot for remote maintenance applications in hazardous environments, Jrnl of Robotics and Autonomous Systems, vol. 53/2, pp. 142-52. 2005.

[16] B. Rooks, Plotting future UK robotics research Programmes, IND ROBOT vol. 33 (3), pp. 165-169. 2006.

[17] D.A. Sanders, GE Tewkesbury and DC Robinson, Simple expert systems to improve an ultrasonic sensor-system for a tele-operated mobile-robot. SENSOR REV, vol. 31, no. 3, pp. 246-260. 2011.

[18] D.A Sanders and I.J. STOTT, Analysis of failure rates with a teleoperated mobile robot between a human tele-operator and a human with a sensor system to assist. Robotica Journal. 2011.

[19] D Sanders, G. Tewkesbury and J. Graham-Jones,Simple rules to modify pre-planned paths to improve gross robot motions associated with pick \& place assembly tasks. ASSEMBLY AUTOM, vol. 31 (1) 2011.

[20] D.A. Sanders, Comparing ability to complete simple tele-operated rescue or maintenance mobile-robot tasks with and without a sensor system. SENSOR REV, vol. 30, no. 1, pp. 40-50. 2010.

[21] D.A. Sanders, J. Graham-Jones and A. Gegov, Improving ability of teleoperators to complete progressively more difficult mobile robot paths using simple expert systems and ultrasonic sensors. IND ROBOT, vol. 37, no. 5, pp. 431-440. 2010.

[22] I. Stott and D. Sanders, New powered wheelchair systems for the rehabilitation of some severely disabled users, Int Jrnl REHAB RESEARCH vol. 23 (3), pp. 149-153. 2000.

[23] D. Sanders and I. Stott, A new prototype intelligent mobility system to assist powered wheelchair users IND ROBOT vol. 26 (6), pp. 466-475. 1999.

[24] M.J Goodwin, D.A. Sanders and G.A. Poland et al, Navigational assistance for disabled wheelchair-users, proc' of Euromicro Conference 95 vol. 43, pp. 73-79. 1997.

[25] D. Sanders, Analysis of the effects of time delays on the teleoperation of a mobile robot in various modes of operation, IND ROBOT vol. 36 (6) pp. 570-584. 2009.

[26] D. Sanders, I. Stott, J Graham-Jones et al, Expert system to interpret hand tremor and provide joystick position signals for powered wheelchairs with ultrasonic sensor systems, IND ROBOT vol. 38 (6), pp. 585-598. 2011.

[27] D.A. Sanders, N. Bausch, H. Liu, et al, Improving Steering of a Powered Wheelchair Using an Expert System to Interpret Hand Tremor, Proc' Intelligent Robotics \& Applications, Pt I vol. 9245 pp. 460-471. 2015.

[28] D. Sanders and I. Stott, The use of virtual reality to train powered wheelchair users and test new wheelchair systems, International Journal of Rehabilitation Research vol. 23 (4), pp. 321-326. 2000.

[29] D. Sands, Cost effective robotics in the nuclear industry. IND ROBOT, vol. 33, No 3, pp 170 - 173. 2006.

[30] T. Nakamura and K. Satoh, Development of an omni-directional mobile robot using traveling waves based on snail locomotion. IND ROBOT, vol. 35 (3), pp. 206-210. 2008.

[31] B.L Luk, A.A. Collie, D.S Cooke and S.Chen Walking and climbing service robots for safety inspection of nuclear reactor pressure vessels. Measurement \& Control vol. 39 (2), pp. 43-47. 2006.

[32] B. Luk, K. Liu, A. Collie, D. Cooke \& S. Chen, Tele-operated climbing and mobile service robots for remote inspection and maintenance in nuclear industry, IND ROBOT, vol. 33, No 3, pp. 194 - 204. 2006.

[33] M.J Bakari and D.W Seward, Human arm-like mechanical manipulator the design and development of a multi-arm mobile robot for nuclear decommissioning. Proc' 3rd Int, Conf Informatics in control, automation and robotics - Robotics and Automation, pp. 168-175. 2006.

[34] B.P DeJong E.L Faulring, J Edward Colgate, M.A Peshkin, H. Kang, Y.S Park and T.F Ewing, Lessons learned from a novel tele-operation test-bed. . IND ROBOT, vol. 33, No 3, pp. 187 - 193. 2006.

[35] D. A. Sanders, I. J. Stott, D. C. Robinson, et al., "Analysis of successes and failures with a tele-operated mobile robot in various modes of 
operation", Robotica, vol. 30, pp. 973-988, 2012.

[36] I. J. Stott and D. A. Sanders, "New powered mobile robot systems for the rehabilitation of some severely disabled users", Int Jrnl of Rehab Research, vol. 23, no. 3, pp. 149-153, 2000.

[37] D. A. Sanders, J. Graham-Jones, and A. Gegov, "Improving ability of tele-operators to complete progressively more difficult mobile robot paths using simple expert systems and ultrasonic sensors", Industrial Robot-an International Journal, vol. 37, no. 5, pp. 431-440, 2010.

[38] D. A. Sanders and I. J. Stott, "A new prototype intelligent mobility system to assist powered mobile robot users", Industrial Robot, vol. 26,no. 6, pp. 466-475, 1999.

[39] K. Kuniaki, I. Tatsuya, F. Teruo, N. Takashi, A. Asama and E. Isao, "Teleoperation of Autonomous Mobile Robot under Limited Feedback Information," in Proc. of International Conf on Field and Service Robotics, London, United Kingdom, 1998, pp 146-151.

[40] D. G. Macharet and D. A. Florencio, "A Collaborative Control System for Telepresence Robots," in Proc. of IEEE/RSJ International Conf on Intelligent Robots and Systems, Algarve, Portugal, 2012, pp. 5105-5111.

[41] S. Hayati and S. T. Venkataraman, "Design and Implementation of a Robot Control System with Traded and Shared Control Capability," in Proc. of IEEE International Conf on Robotics and Automation, Scottsdal, AZ, USA, 1989, pp. 1310-1315.

[42] A. David M. M. Abbink, and E. R. Boer, "Haptic Shared Control: Smoothly Shifting Control Authority?," Cognition, Technology \& Work, Springer-Verlag, vol. 14, no. 1, pp. 19-28, 2012.

[43] Makoto Itoh, Toshiyuki Inagakia and Hiroto Tanaka, "Haptic Steering Direction Guidance for Pedestrian-Vehicle Collision Avoidance," in Proc. of IEEE Int Conf on System, Man, and Cybernetics, Seoul, Korea, 2012, pp. 3309-3314.

[44] T. Carlson and Y. Demiris, "Increasing Robotic Mobile robot Safety With Collaborative Control: Evidence from Secondary Task Experiments," in Proc. of IEEE International Conf on Robotics and Automation, Anchorage, Alaska, USA, 2010, pp. 5582-5587.

[45] A. R. Satti, D. Coyle and G. Prasad, "Self-paced Brain-controlled Mobile robot Methodology with Shared and Automated Assistive Control," in Proc. of IEEE Symposium on Computational Intelligence, Cognitive Algorithms, Mind, and Brain, Paris, France, 2011, pp. 1-8.

[46] A. Franchi, C. Secchi, M. Ryll, H. H. Bulthoff and P. R. Giordano, "Shared Control : Balancing Autonomy and Human Assistance with a Group of Quadrotor UAVs," IEEE Robotics \& Automation Magazine, vol.19, no.3, pp. 57-68, 2012.

[47] L. Tonin, R. Leeb, M. Tavella, S. Perdikis and J. del R. Millan, "The Role of Shared-Control in BCI-based Telepresence," in Proc. of IEEE International Conf on Systems, Man, and Cybernetics, Istanbul, Turkey, 2010, pp. 1462 - 1466

[48] H. K. Kim, S. J. Biggs, D. W. Schloerb, J. M. Carmena, M. A. Lebedev, M. A. L. Nicolelis and M. A. Srinivasan, "Continuous Shared Control for Stabilizing Reaching and Grasping With Brain-Machine Interfaces," IEEE Transactions on Biomedical Engineering, vol. 53, no. 6, pp. 1164-1173. 2006.

[49] D.A. Sanders, S.D. Urwin-Wright, G.E. Tewkesbury, GE; et al. "Pointer device for thin-film transistor and cathode ray tube computer screens", Electronics Letters, vol. 41, no. 16, 2005, pp. 894-896.

[50] D.A. Sanders, "Analysis of the effects of time delays on the teleoperation of a mobile robot in various modes of operation", Ind Robot vol. 36, no. 6, 2009, pp. 570-584.

[51] D.A. Sanders, "Controlling the direction of "walkie" type forklifts and pallet jacks on sloping ground", Assembly Automation, vol. 28, no. 4, 2008, pp. 317-324.

[52] D.A. Sanders and J Bergasa-Suso, "Inferring Learning Style From the Way Students Interact With a Computer User Interface and the WWW", IEEE Transactions on Education vol. 53, no. 4, 2010, pp. 613-620.

[53] D.A. Sanders and G.E Tewkesbury, "A pointer device for TFT display screens that determines position by detecting colours on the display using a colour sensor and an Artificial Neural Network", Displays, vol. 30, no. 2, 2009, pp. 84-96.

[54] D.A. Sanders and A Baldwin, "X-by-wire technology", Total Vehicle Technology Conference, ISBN:1-86058-324-5, pp. 3-12. 2001.
[55] D.A. Sanders, "Comparing ability to complete simple tele-operated rescue or maintenance mobile-robot tasks with and without a sensor system”, Sensor Review vol. 30, no. 1, 2010, pp. 40-50.

[56] T. Carlson and Y. Demiris, "Collaborative Control for a Robotic Mobile robot: Evaluation of Performance, Attention, and Workload," IEEE Trans. on Systems, Man, and Cybernetics-Part B: Cybernetics, vol. 42, no. 3, 2012, pp. 876-888.

[57] J. Kim, D. Chang, H. Ladjal, D. Folio, A. Ferreira and J. Kim, "Evaluation of Telerobotic Shared Control for Efficient Manipulation of Single-Cells in Microinjection," in Proc. of IEEE Int Conf on Robotics and Automation, Shanghai, China, 2011, pp. 3382-3387.

[58] Q. Li, W. Chen and J. Wang, "Dynamic Shared Control for Human-Mobile robot Cooperation," in Proc of IEEE International Conf on Robotics and Automation, Shanghai, China, 2011, pp. 42784283

[59] T. Carlson, R. Leeb, R. Chavarriage and J. del R. Millan, "Online Modulation of the Level of Assistance in Shared Control System," in Proc of IEEE Int Conf on System, Man, and Cybernetics, Seoul, Korea, 2012, pp. 3321-3326.

[60] S. Y. Jiang and K-T. Song, "Differential Flatness-based Motion Control of a Steer-and-Drive Omnidirectional Mobile Robot," in Proc. of IEEE Int Conf on Mechatronics and Automation, Kagawa, Japan, 2013, pp. 1167-1172.

[61] D. A. Sanders, "Using Confidence factors to decide how to share control between human powered wheelchair drivers and ultrasonic sensors", IEEE Transactions on Neural Systems and Rehabilitation Engineering DOI: 10.1109/TNSRE.2016.2620988. 